\title{
ON THE RESCALED RIEMANNIAN METRIC OF CHEEGER GROMOLL TYPE ON THE COTANGENT BUNDLE
}

\author{
A. GEZER AND M. ALTUNBAS
}

\begin{abstract}
Let $(M, g)$ be an $n$-dimensional Riemannian manifold and $T^{*} M$ be its cotangent bundle equipped with a Riemannian metric of Cheeger Gromoll type which rescale the horizontal part by a nonzero differentiable function. The main purpose of the present paper is to discuss curvature properties of $T^{*} M$ and construct almost paracomplex Norden structures on $T^{*} M$. We investigate conditions for these structures to be para-Kähler (paraholomorphic) and quasi-Kähler. Also, some properties of almost paracomplex Norden structures in context of almost product Riemannian manifolds are presented.
\end{abstract}

\section{Introduction}

Geometric structures on bundles have been object of much study since the middle of the last century. The natural lifts of the metric $g$, from a Riemannian manifold $(M, g)$ to its tangent or cotangent bundles, induce new (pseudo) Riemannian structures, with interesting geometric properties. Maybe the best known Riemannian metric ${ }^{S} g$ on the tangent bundle over Riemannian manifold $(M, g)$ is that introduced by Sasaki in 1958 (see [29]), but in most cases the study of some geometric properties of the tangent bundle endowed with this metric led to the flatness of the base manifold. This metric ${ }^{S} g$ is a standard notion in differential geometry called the Sasaki metric. The Sasaki metric ${ }^{S} g$ has been extensively studied by several authors and in many different contexts. Another Riemannian metric ${ }^{C G} g$ on the tangent bundle $T M$ had been defined, some years before, by E. Musso and F. Tricerri [16] who, inspired by the paper 44 of J. Cheeger and D. Gromoll, called it the Cheeger-Gromoll metric. The metric was defined by J. Cheeger and D. Gromoll; yet, there were E. Musso and F. Tricerri who wrote down its expression, constructed it in a more "comprehensible" way, and gave it the name. In 38 (see also [39, 40, B. V. Zayatuev introduced a Riemannian metric ${ }^{S} \bar{g}$ on the tangent bundle $T M$ given by

$$
\begin{aligned}
{ }^{S} \bar{g}\left({ }^{H} X,{ }^{H} Y\right) & =f g(X, Y), \\
{ }^{S} \bar{g}\left({ }^{H} X,{ }^{V} Y\right) & ={ }^{S} \bar{g}\left({ }^{V} X,{ }^{H} Y\right)=0, \\
{ }^{S} \bar{g}\left({ }^{V} X,{ }^{V} Y\right) & =g(X, Y),
\end{aligned}
$$

where $f>0, f \in C^{\infty}(M)$. For $f=1$, it follows that ${ }^{S} \bar{g}={ }^{S} g$, i.e. the metric ${ }^{S} \bar{g}$ is a generalization of the Sasaki metric ${ }^{S} g$. Also, the authors studied the rescaled

2000 Mathematics Subject Classification. 53C07, 53C55, 55R10.

Key words and phrases. Almost paracomplex structure, connection, cotangent bundle, paraholomorphic tensor field, Riemannian metric. 
Sasaki type metric on the cotangent bundle $T^{*} M$ over Riemannian manifold $(M, g)$ (see [10]).

Almost complex Norden and almost paracomplex Norden structures are among the most important geometrical structures which can be considered on a manifold. Let $M_{2 k}$ be a $2 k$-dimensional differentiable manifold endowed with an almost (para) complex structure $\varphi$ and a pseudo-Riemannian metric $g$ of signature $(k, k)$ such that $g(\varphi X, Y)=g(X, \varphi Y)$, i.e. $g$ is pure with respect to $\varphi$ for arbitrary vector fields $X$ and $Y$ on $M_{2 k}$. Then the metric $g$ is called Norden metric. Norden metrics are referred to as anti-Hermitian metrics or $B$-metrics. They find widespread application in mathematics as well as in theoretical physics. Many authors considered almost (para)complex Norden structures on the tangent, cotangent and tensor bundles $[7,9,18,19,20,21,22,23,24,26,27$.

Let $T^{*} M$ be the cotangent bundle of a Riemannian manifold $(M, g)$. We define $r^{2}=g^{-1}(p, p)=g^{i j} p_{i} p_{j}$ and put $\alpha=1+r^{2}$. Then the rescaled Riemannian metric of Cheeger Gromoll type ${ }^{C G} g_{f}$ is defined on $T^{*} M$ by the following three equations

$$
\begin{gathered}
{ }^{C G} g_{f}\left({ }^{V} \omega,{ }^{V} \theta\right)=\frac{1}{\alpha}\left(g^{-1}(\omega, \theta)+g^{-1}(\omega, p) g^{-1}(\theta, p)\right), \\
{ }^{C G} g_{f}\left({ }^{V} \omega,{ }^{H} Y\right)=0 \\
{ }^{C G} g_{f}\left({ }^{H} X,{ }^{H} Y\right)=f g(X, Y)
\end{gathered}
$$

for any $X, Y \in \Im_{0}^{1}(M)$ and $\omega, \theta \in \Im_{1}^{0}(M)$, where $f>0, f \in C^{\infty}(M), g^{-1}(\omega, \theta)=$ $g^{i j} \omega_{i} \theta_{j}$ for all $\omega, \theta \in \Im_{1}^{0}(M)$ (for $f=1$, see [2]). In this paper, firstly, curvature tensor of the rescaled Cheeger Gromoll type metric ${ }^{C G} g_{f}$ are presented. Secondly, we get the conditions under which the cotangent bundle endowed with some paracomplex structures and the rescaled Riemannian metric of Cheeger Gromoll type ${ }^{C G} g_{f}$ is a paraholomorphic Norden manifold. Finally, for an almost paracomplex manifold to be an specialized almost product manifold, we give some results relation to Riemannian almost product structure on the cotangent bundle.

Throughout this paper, all manifolds, tensor fields and connections are always assumed to be differentiable of class $C^{\infty}$. Also, we denote by $\Im_{q}^{p}(M)$ the set of all tensor fields of type $(p, q)$ on $M$, and by $\Im_{q}^{p}\left(T^{*} M\right)$ the corresponding set on the cotangent bundle $T^{*} M$. The Einstein summation convention is used, the range of the indices $i, j, s$ being always $\{1,2, \ldots, n\}$.

\section{Preliminaries}

2.1. The cotangent bundle. The cotangent bundle of a smooth $n$-dimensional Riemannian manifold may be endowed with a structure of $2 n$-dimensional smooth manifold, induced by the structure on the base manifold. If $(M, g)$ is a smooth Riemannian manifold of dimension $n$, we denote its cotangent bundle by $\pi: T^{*} M \rightarrow$ $M$. A system of local coordinates $\left(U, x^{i}\right), i=1, \ldots, n$ in $M$ induces on $T^{*} M$ a system of local coordinates $\left(\pi^{-1}(U), x^{i}, x^{\bar{i}}=p_{i}\right), \bar{i}=n+i=n+1, \ldots, 2 n$, where $x^{\bar{i}}=p_{i}$ is the components of covectors $p$ in each cotangent space $T_{x}^{*} M, x \in U$ with respect to the natural coframe $\left\{d x^{i}\right\}$.

Let $X=X^{i} \frac{\partial}{\partial x^{i}}$ and $\omega=\omega_{i} d x^{i}$ be the local expressions in $U$ of a vector field $X$ and a covector (1-form) field $\omega$ on $M$, respectively. Then the vertical lift ${ }^{V} \omega$ of $\omega$ 
and the horizontal lift ${ }^{H} X$ of $X$ are given, with respect to the induced coordinates, by

$$
V_{\omega}=\omega_{i} \partial_{\bar{i}}
$$

and

$$
{ }^{H} X=X^{i} \partial_{i}+p_{h} \Gamma_{i j}^{h} X^{j} \partial_{\bar{i}},
$$

where $\partial_{i}=\frac{\partial}{\partial x^{i}}, \partial_{\bar{i}}=\frac{\partial}{\partial x^{\bar{i}}}$ and $\Gamma_{i j}^{h}$ are the coefficients of the Levi-Civita connection $\nabla$ of $g$.

The Lie bracket operation of vertical and horizontal vector fields on $T^{*} M$ is given by the formulas

$$
\left\{\begin{array}{l}
{\left[{ }^{H} X,{ }^{H} Y\right]={ }^{H}[X, Y]+{ }^{V}(p \circ R(X, Y))} \\
\left.{ }^{H} X,{ }^{V} \omega\right]={ }^{V}\left(\nabla_{X} \omega\right) \\
\left.{ }^{V} \theta,{ }^{V} \omega\right]=0
\end{array}\right.
$$

for any $X, Y \Im_{0}^{1}(M)$ and $\theta, \omega \in \Im_{1}^{0}(M)$, where $R$ is the Riemannian curvature of $g$ defined by $R(X, Y)=\left[\nabla_{X}, \nabla_{Y}\right]-\nabla_{[X, Y]}$ (for details, see [36]).

2.2. Expressions in the adapted frame. We insert the adapted frame which allows the tensor calculus to be efficiently done in $T^{*} M$. With the connection $\nabla$ of $g$ on $M$, we can introduce adapted frames on each induced coordinate neighborhood $\pi^{-1}(U)$ of $T^{*} M$. In each local chart $U \subset M$, we write $X_{(j)}=\frac{\partial}{\partial x^{j}}, \theta^{(j)}=d x^{j}, j=$ $1, \ldots, n$. Then from (2.1) and (2.2), we see that these vector fields have, respectively, local expressions

$$
\begin{gathered}
{ }^{H} X_{(j)}=\frac{\partial}{\partial x^{j}}+p_{a} \Gamma_{h j}^{a} \partial_{\bar{h}} \\
{ }^{V} \theta^{(j)}=\frac{\partial}{\partial x^{\bar{j}}}
\end{gathered}
$$

with respect to the natural frame $\left\{\frac{\partial}{\partial x^{j}}, \frac{\partial}{\partial x^{\bar{j}}}\right\}$. These $2 n$ vector fields are linearly independent and they generate the horizontal distribution of $\nabla_{g}$ and the vertical distribution of $T^{*} M$, respectively. We call the set $\left\{{ }^{H} X_{(j)},{ }^{V} \theta^{(j)}\right\}$ the frame adapted to the connection $\nabla$ of $g$ in $\pi^{-1}(U) \subset T^{*} M$. By denoting

$$
\begin{aligned}
& E_{j}={ }^{H} X_{(j)}, \\
& E_{\bar{j}}={ }^{V} \theta^{(j)},
\end{aligned}
$$

we can write the adapted frame as $\left\{E_{\alpha}\right\}=\left\{E_{j}, E_{\bar{j}}\right\}$. The indices $\alpha, \beta, \gamma, \ldots=$ $1, \ldots, 2 n$ indicate the indices with respect to the adapted frame.

Using (2.1), (2.2) and (2.4), we have

$$
V_{\omega}=\left(\begin{array}{l}
0 \\
\omega_{j}
\end{array}\right),
$$

and

$$
{ }^{H} X=\left(\begin{array}{l}
X^{j} \\
0
\end{array}\right)
$$


with respect to the adapted frame $\left\{E_{\alpha}\right\}$ (for details, see [36]). By the straightforward calculations, we have the lemma below.

Lemma 1. The Lie brackets of the adapted frame of $T^{*} M$ satisfy the following identities:

$$
\begin{aligned}
{\left[E_{i}, E_{j}\right] } & =p_{s} R_{i j l}^{s} E_{\bar{l}}, \\
{\left[E_{i}, E_{\bar{j}}\right] } & =\Gamma_{i l}^{j} E_{\bar{l}}, \\
{\left[E_{\bar{i}}, E_{\bar{j}}\right] } & =0
\end{aligned}
$$

where $R_{i j l}{ }^{s}$ denote the components of the curvature tensor of $(M, g)$.

\section{Curvature tensor of the Rescaled Riemannian metric of Cheeger}

\section{GROMOLL TYPE}

From the equations (1.1)-(1.3), by virtue of (2.5) and (2.6), the rescaled Cheeger Gromoll type metric ${ }^{C G} g_{f}$ has components with respect to the adapted frame $\left\{E_{\alpha}\right\}$ :

$$
{ }^{C G} g_{f}=\left(\begin{array}{cc}
f g_{i j} & 0 \\
0 & \frac{1}{\alpha}\left(g^{i j}+g^{i s} g^{t j} p_{s} p_{t}\right)
\end{array}\right) .
$$

For the Levi-Civita connection of the rescaled Cheeger Gromoll type metric ${ }^{C G} g_{f}$ we give the next theorem.

Theorem 1. Let $(M, g)$ be a Riemannian manifold and equip its cotangent bundle $T^{*} M$ with the rescaled Cheeger Gromoll type metric ${ }^{C G} g_{f}$. Then the corresponding Levi-Civita connection $\widetilde{\nabla}$ satisfies the followings:

$$
\left\{\begin{array}{l}
\text { i) } \widetilde{\nabla}_{E_{i}} E_{j}=\left\{\Gamma_{i j}^{l}+{ }^{f} A_{i j}^{l}\right\} E_{l}+\frac{1}{2} p_{s} R_{i j l}{ }^{s} E_{\bar{l}}, \\
\text { ii) } \widetilde{\nabla}_{E_{i}} E_{\bar{j}}=\frac{1}{2 f \alpha} p_{s} R_{. i}^{l}{ }^{j s} E_{l}-\Gamma_{i l}^{j} E_{\bar{l}}, \\
\text { iii) } \widetilde{\nabla}_{E_{\bar{i}}} E_{j}=\frac{1}{2 f \alpha} p_{s} R_{\cdot j}^{l}{ }^{i s} E_{l}, \\
\text { iv) } \widetilde{\nabla}_{E_{\bar{i}}} E_{\bar{j}}=\left\{\frac{-1}{\alpha}\left(p^{i} \delta_{l}^{j}+p^{j} \delta_{l}^{i}\right)+\frac{\alpha+1}{\alpha^{2}} g^{i j} p_{l}+\frac{1}{\alpha^{2}} p^{i} p^{j} p_{l}\right\} E_{\bar{l}}
\end{array}\right.
$$

with respect to the adapted frame, where ${ }^{f} A_{j i}^{h}$ is a tensor field of type $(1,2)$ defined by ${ }^{f} A_{j i}^{h}=\frac{1}{f}\left(f_{j} \delta_{i}^{h}+f_{i} \delta_{j}^{h}-f_{.}^{m} g_{j i}\right)$ and $p^{i}=g^{i t} p_{t}, R_{. j}^{k}{ }^{i s}=g^{k t} g^{i m} R_{t j m}$.

Proof. The connection $\widetilde{\nabla}$ is characterized by the Koszul formula:

$$
\begin{aligned}
2^{C G} g_{f}(\widetilde{\nabla} \widetilde{X} \widetilde{Y}, \widetilde{Z}) & =\widetilde{X}\left({ }^{C G} g_{f}(\widetilde{Y}, \widetilde{Z})\right)+\widetilde{Y}\left({ }^{C G} g_{f}(\widetilde{Z}, \widetilde{X})\right)-\widetilde{Z}\left({ }^{C G} g_{f}(\widetilde{X}, \widetilde{Y})\right) \\
-{ }^{C G} g_{f}(\widetilde{X},[\widetilde{Y}, \widetilde{Z}]) & +{ }^{C G} g_{f}(\widetilde{Y},[\widetilde{Z}, \widetilde{X}])+{ }^{C G} g_{f}(\widetilde{Z},[\widetilde{X}, \widetilde{Y}])
\end{aligned}
$$

for all vector fields $\widetilde{X}, \widetilde{Y}$ and $\widetilde{Z}$ on $T^{*} M$. One can verify the Koszul formula for pairs $\widetilde{X}=E_{i}, E_{\bar{i}}$ and $\widetilde{Y}=E_{j}, E_{\bar{j}}$ and $\widetilde{Z}=E_{k}, E_{\bar{k}}$. In calculations, the formulas (2.4) Lemma 1 and the first Bianchi identity for $R$ should be applied. We omit standart calculations. 
Let $\tilde{X}, \tilde{Y} \in \Im_{0}^{1}\left(T^{*} M\right)$. Then the covariant derivative $\tilde{\nabla}_{\tilde{Y}} \tilde{X}$ has components

$$
\tilde{\nabla}_{\tilde{Y}} \tilde{X}^{\alpha}=\tilde{Y}^{\gamma} E_{\gamma} \tilde{X}^{\alpha}+\tilde{\Gamma}_{\gamma \beta}^{\alpha} \tilde{X}^{\beta} \tilde{Y}^{\gamma}
$$

with respect to the adapted frame $\left\{E_{\alpha}\right\}$. Using (2.4), (2.5), (2.6) and (3.2), we have the following proposition.

Proposition 1. Let $(M, g)$ be a Riemannian manifold and $\widetilde{\nabla}$ be the Levi-Civita connection of the cotangent bundle $T^{*} M$ equipped with the rescaled Cheeger Gromoll type metric ${ }^{C G} g_{f}$. Then

i) $\widetilde{\nabla}_{H X}{ }^{H} Y={ }^{H}\left(\nabla_{X} Y+{ }^{f} A(X, Y)\right)+\frac{1}{2} V(p \circ R(X, Y))$,

ii) $\widetilde{\nabla}_{H}{ }^{V} \theta=\frac{1}{2 f \alpha}{ }^{H}\left(p\left(g^{-1} \circ R(, X) \widetilde{\theta}\right)\right)+{ }^{V}\left(\nabla_{X} \theta\right)$,

iii) $\widetilde{\nabla}_{V_{\omega}}{ }^{H} Y=\frac{1}{2 f \alpha}^{H}\left(p\left(g^{-1} \circ R(, Y) \tilde{\omega}\right)\right)$,

iv) $\widetilde{\nabla}_{{ }}{ }^{V} \theta=-\frac{1}{\alpha}\left({ }^{C G} g\left({ }^{V} \omega, \gamma \delta\right){ }^{V} \theta+{ }^{C G} g_{f}\left({ }^{V} \theta, \gamma \delta\right){ }^{V} \omega\right)+\frac{\alpha+1}{\alpha}{ }^{C G} g_{f}\left({ }^{V} \omega,{ }^{V} \theta\right) \gamma \delta$ $-\frac{1}{\alpha}{ }^{C G} g_{f}\left({ }^{V} \omega, \gamma \delta\right)^{C G} g_{f}\left({ }^{V} \theta, \gamma \delta\right) \gamma \delta$

for all $X, Y \in \Im_{0}^{1}(M), \omega, \theta \in \Im_{1}^{0}(M)$, where $\tilde{\omega}=g^{-1} \circ \omega \in \Im_{0}^{1}(M), R(, X) \tilde{\omega} \in$ $\Im_{1}^{1}(M), g^{-1} \circ R(, X) \tilde{\omega} \in \Im_{0}^{1}(M), R$ and $\gamma \delta$ denote respectively the curvature tensor of $\nabla$ and the canonical or Liouville vector field on $T^{*} M$ with the local expression $\gamma \delta=p_{i} E_{\bar{i}}($ for $f=1$, see [2]).

The Riemannian curvature tensor $\widetilde{R}$ of $T^{*} M$ with the rescaled Cheeger Gromoll type metric ${ }^{C G} g_{f}$ is obtained from the well-known formula

$$
\widetilde{R}(\widetilde{X}, \widetilde{Y}) \widetilde{Z}=\widetilde{\nabla}_{\widetilde{X}} \widetilde{\nabla}_{\widetilde{Y}} \widetilde{Z}-\widetilde{\nabla}_{\widetilde{Y}} \widetilde{\nabla}_{\widetilde{X}} \widetilde{Z}-\widetilde{\nabla}_{[\widetilde{X}, \widetilde{Y}]} \widetilde{Z}
$$

for all $\tilde{X}, \widetilde{Y}, \widetilde{Z} \in \Im_{0}^{1}\left(T^{*} M\right)$. Then from Lemma 1 and Theorem 1, we get the following proposition.

Proposition 2. The components of the curvature tensor $\widetilde{R}$ of of the cotangent bundle $T^{*} M$ with the rescaled Cheeger Gromoll type metric ${ }^{C G} g_{f}$ are given as follows:

$$
\begin{aligned}
& \widetilde{R}\left(E_{l}, E_{i}\right) E_{j}=\left\{R_{l i j}^{m}-\frac{1}{2 f \alpha} p_{t} p_{a} R_{l i h}^{a} R_{. j}^{m}{ }^{h t}+\frac{1}{4 f \alpha} p_{t} p_{a}\left(R_{.}^{m}{ }_{l}{ }^{h t}{ }^{h t} R_{i j h}^{a}-R_{.}^{m}{ }_{i}{ }^{h t} R_{l j h}^{a}\right)\right. \\
& \left.\left.+\nabla_{l}\left(A_{i j}^{m}\right)-\nabla_{i}\left(A_{l j}^{m}\right)+A_{l h}^{m} A_{i j}^{h}-A_{i h}^{m} A_{l j}^{h}\right)\right\} E_{m} \\
& +\left\{\frac{1}{2 f} p_{t}\left(\nabla_{l} R_{i j m}^{t}-\nabla_{i} R_{l j m}^{t}\right)+\frac{1}{2} p_{t}\left(R_{l h m}^{t} A_{i j}^{h}-R_{i h m}^{t} A_{l j}^{h}\right)\right\} E_{\bar{m}} \\
& \widetilde{R}\left(E_{\bar{l}}, E_{i}\right) E_{j}=\left\{\frac{-1}{2 f \alpha} p_{a} \nabla_{i} R_{. j}^{m}{ }_{j}^{l a}+\frac{1}{2 f \alpha} p_{a}\left(R_{., h^{m} .} A_{i j}^{h}-R_{. j}^{h{ }^{l a}} A_{i h}^{m}+\frac{f_{i}}{f} R_{. j}^{m}{ }_{j}{ }^{l a}\right)\right\} E_{m} \\
& +\left\{\frac{1}{2} R_{i j m}^{l}-\frac{1}{4 f \alpha} p_{t} p_{a} R_{i h m}^{t} R_{. j}^{h}{ }_{.}^{l a}-\frac{1}{2 \alpha} p_{a} p^{l} R_{i j m}^{a}-\frac{\alpha+1}{2 \alpha^{2}} p_{a} p_{m} R_{i j}{ }^{l a}\right\} E_{\bar{m}}
\end{aligned}
$$

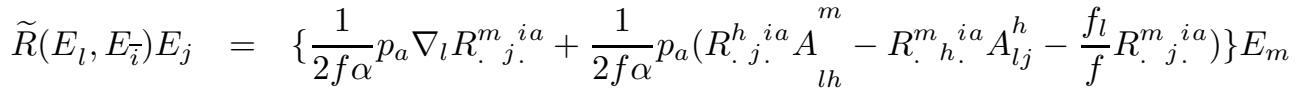

$$
\begin{aligned}
& +\left\{\frac{-1}{2} R_{l j m}^{i}-\frac{1}{4 f \alpha} p_{t} p_{a} R_{l h m}^{a} R_{. j}^{h}{ }^{i t}+\frac{1}{2 \alpha} p_{a} p^{i} R_{l j m}^{a}-\frac{\alpha+1}{2 \alpha^{2}} p_{a} p_{m} R_{l j}{ }^{i a}\right\} E_{\bar{m}}
\end{aligned}
$$

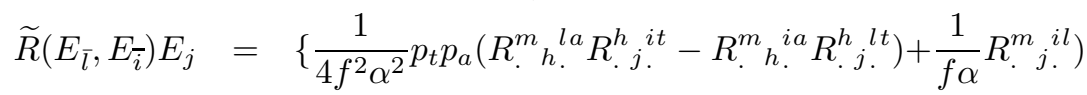

$$
\begin{aligned}
& +\frac{1}{f \alpha^{2}} p_{a}\left(p^{i} R_{\cdot{ }^{m}{ }^{l a}-}-p^{l} R_{\cdot{ }^{m}{ }^{i a}}{ }^{i a}\right\} E_{m}
\end{aligned}
$$




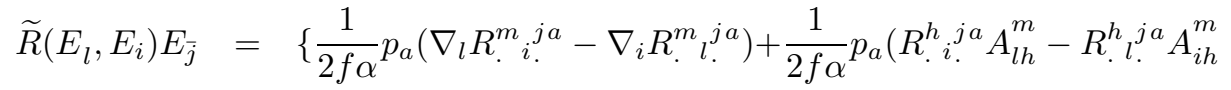

$$
\begin{aligned}
& \left.\left.-\frac{f_{l}}{f} R_{\cdot{ }^{m}{ }^{j a} \cdot}+\frac{f_{i}}{f} R_{\cdot{ }^{m} l^{j a}}^{j a}\right)\right\} E_{m}+\left\{R_{i l m}^{j}+\frac{1}{4 f \alpha} p_{t} p_{a}\left(R_{l h m}^{t} R_{\cdot i \cdot}^{h{ }^{j a}}\right.\right. \\
& \left.\left.-R_{i h m}^{a} R_{.}^{h} l^{j t}\right)+\frac{1}{\alpha} p_{a} p^{j} R_{l i m}^{a}-\frac{\alpha+1}{\alpha^{2}} p_{a} p_{m} R_{l i}{ }^{j a}\right\} E_{\bar{m}}
\end{aligned}
$$

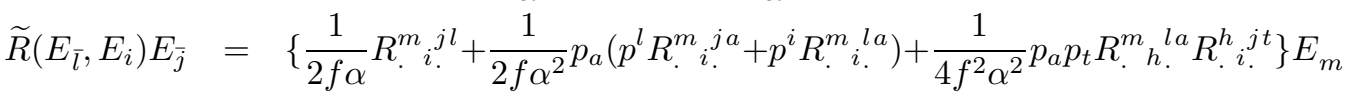

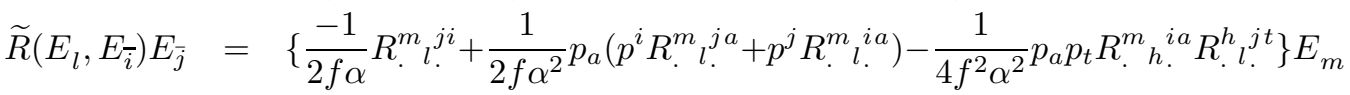

$$
\begin{aligned}
& \widetilde{R}\left(E_{\bar{l}}, E_{\bar{i}}\right) E_{\bar{j}}=\left\{\frac{\alpha^{2}+\alpha+1}{\alpha^{3}}\left(g^{i j} \delta_{m}^{l}-g^{j l} \delta_{m}^{i}\right)+\frac{\alpha+2}{\alpha^{3}}\left(g^{l j} p^{i} p_{m}-g^{i j} p_{l} p_{m}\right)\right. \\
& \left.+\frac{\alpha-1}{\alpha^{3}}\left(\delta_{m}^{i} p^{l} p^{j}-\delta_{m}^{l} p^{i} p^{j}\right)\right\} E_{\bar{m}}
\end{aligned}
$$

with respect to the adapted frame $\left\{E_{\alpha}\right\}$ (for $f=1$, see [2]).

\section{4. para-Kähler (or paraholomorphic) Norden structures on $T^{*} M$}

An almost paracomplex manifold is an almost product manifold $\left(M_{2 k}, \varphi\right), \varphi^{2}=$ $i d, \varphi \neq \pm i d$ such that the two eigenbundles $T^{+} M_{2 k}$ and $T^{-} M_{2 k}$ associated to the two eigenvalues +1 and -1 of $\varphi$, respectively, have the same rank. Note that the dimension of an almost paracomplex manifold is necessarily even. This structure is said to be integrable if the matrix $\varphi=\left(\varphi_{j}^{i}\right)$ is reduced to the constant form in a certain holonomic natural frame in a neighborhood $U_{x}$ of every point $x \in M_{2 k}$. On the other hand, an almost paracomplex structure is integrable if and only if one can introduce a torsion-free linear connection such that $\nabla \varphi=0$. A paracomplex manifold is an almost paracomplex manifold $\left(M_{2 k}, \varphi\right)$ such that the G-structure defined by the affinor field $\varphi$ is integrable. Also it can be give another-equivalentdefinition of paracomplex manifold in terms of local homeomorphisms in the space $R^{k}(j)=\left\{\left(X^{1}, \ldots, X^{k}\right) / X^{i} \in R(j), i=1, \ldots, k\right\}$ and paraholomorphic changes of charts in a way similar to [5] (see also [34]), i.e. a manifold $M_{2 k}$ with an integrable paracomplex structure $\varphi$ is a realization of the paraholomorphic manifold $M_{k}(R(j))$ over the algebra $R(j)$.

A tensor field $\omega$ of type $(0, q)$ is called pure tensor field with respect to $\varphi$ if

$$
\omega\left(\varphi X_{1}, X_{2}, \ldots, X_{q}\right)=\omega\left(X_{1}, \varphi X_{2}, \ldots, X_{q}\right)=\ldots=\omega\left(X_{1}, X_{2}, \ldots, \varphi X_{q}\right)
$$

for any $X_{1}, \ldots, X_{q} \in \Im_{0}^{1}\left(M_{2 k}\right)$. The real model of a paracomplex tensor field $\stackrel{*}{\omega}$ on $M_{k}(R(j))$ is a $(0, q)$-tensor field on $M_{2 k}$ which being pure with respect to $\varphi$. Consider an operator $\Phi_{\varphi}: \Im_{q}^{0}\left(M_{2 k}\right) \rightarrow \Im_{q+1}^{0}\left(M_{2 k}\right)$ applied to the pure tensor field $\omega$ by (see [35])

$$
\begin{gathered}
\left(\Phi_{\varphi} \omega\right)\left(X, Y_{1}, Y_{2}, \ldots, Y_{q}\right)=(\varphi X)\left(\omega\left(Y_{1}, Y_{2}, \ldots, Y_{q}\right)\right)-X\left(\omega\left(\varphi Y_{1}, Y_{2}, \ldots, Y_{q}\right)\right) \\
+\omega\left(\left(L_{Y_{1}} \varphi\right) X, Y_{2}, \ldots, Y_{q}\right)+\ldots+\omega\left(Y_{1}, Y_{2}, \ldots,\left(L_{Y_{q}} \varphi\right) X\right),
\end{gathered}
$$

where $L_{Y}$ denotes the Lie differentiation with respect to $Y$. Let $\varphi$ be a (an almost) paracomplex structure on $M_{2 k}$ and $\Phi_{\varphi} \omega=0$, the (almost) paracomplex tensor field $\stackrel{*}{\omega}$ on $M_{k}(R(j))$ is said to be (almost) paraholomorphic (see [13, 32, 35]). Thus a 
(an almost) paraholomorphic tensor field $\stackrel{*}{\omega}$ on $M_{k}(R(j))$ is realized on $M_{2 k}$ in the form of a pure tensor field $\omega$, such that

$$
\left(\Phi_{\varphi} \omega\right)\left(X, Y_{1}, Y_{2}, \ldots, Y_{q}\right)=0
$$

for any $X, Y_{1}, \ldots, Y_{q} \in \Im_{0}^{1}\left(M_{2 k}\right)$. Therefore, the tensor field $\omega$ on $M_{2 k}$ is also called a (an almost) paraholomorphic tensor field.

An almost paracomplex Norden manifold $\left(M_{2 k}, \varphi, g\right)$ is defined to be a real differentiable manifold $M_{2 k}$ endowed with an almost paracomplex structure $\varphi$ and a Riemannian metric $g$ satisfying Nordenian property (or purity condition)

$$
g(\varphi X, Y)=g(X, \varphi Y)
$$

for any $X, Y \in \Im_{0}^{1}\left(M_{2 k}\right)$. Manifolds of this kind are referred to as anti-Hermitian and B-manifolds (see $7,19,20,21,22,23,24,25,33,34$ ). If $\varphi$ is integrable, we say that $\left(M_{2 k}, \varphi, g\right)$ is a paracomplex Norden manifold. A paracomplex Norden manifold $\left(M_{2 k}, \varphi, g\right)$ is a realization of the paraholomorphic manifold $\left(M_{k}(R(j)), \stackrel{*}{g}\right)$, where $\stackrel{*}{g}=(\stackrel{*}{g}), \quad u, v=1, \ldots, k$ is a paracomplex metric tensor field on $M_{k}(R(j))$.

In a paracomplex Norden manifold, a paracomplex Norden metric $g$ is called paraholomorphic if

$$
\left(\Phi_{\varphi} g\right)(X, Y, Z)=0
$$

for any $X, Y, Z \in \Im_{0}^{1}\left(M_{2 k}\right)$. The paracomplex Norden manifold with paraholomorphic Norden metric $\left(M_{2 k}, \varphi, g\right)$ is called a paraholomorphic Norden manifold.

In [25, Salimov and his collaborators have been proven that for an almost paracomplex manifold with Norden metric $g$, the condition $\Phi_{\varphi} g=0$ is equivalent to $\nabla \varphi=0$, where $\nabla$ is the Levi-Civita connection of $g$. By virtue of this point of view, paraholomorphic Norden manifolds are similar to Kähler manifolds.

V. Cruceanu defined in [6] an almost paracomplex structure on $T^{*} M$ as follows:

$$
\begin{aligned}
J\left({ }^{H} X\right) & =-{ }^{H} X \\
J\left({ }^{V} \omega\right) & ={ }^{V} \omega
\end{aligned}
$$

for any $X \in \Im_{0}^{1}(M)$ and $\omega \in \Im_{1}^{0}(M)$. One can easily check that the metric ${ }^{C G} g_{f}$ is pure with respect to the almost paracomplex structure $J$. Hence we state the following theorem.

Theorem 2. Let $(M, g)$ be a Riemannian manifold and $T^{*} M$ be its cotangent bundle equipped with the rescaled Cheeger Gromoll type metric ${ }^{C G} g_{f}$ and the paracomplex structure $J$. The triple $\left(T^{*} M, J,{ }^{C G} g_{f}\right)$ is an almost paracomplex Norden manifold.

We now give conditions for the rescaled Cheeger Gromoll type metric ${ }^{C G} g_{f}$ to be paraholomorphic with respect to the almost paracomplex structure $J$. Using defination of the rescaled Cheeger Gromoll type metric ${ }^{C G} g_{f}$ and the almost paracomplex structure $J$ and by using the fact that ${ }^{V} \omega^{V}\left(g^{-1}(\theta, \sigma)\right)=0$ and ${ }^{H} X^{V}(f g(Y, Z))={ }^{V}(X(f g(Y, Z)))$ we calculate

$$
\begin{aligned}
\left(\Phi_{J}{ }^{C G} g_{f}\right)(\tilde{X}, \tilde{Y}, \tilde{Z}) & =(J \tilde{X})\left({ }^{C G} g_{f}(\tilde{Y}, \tilde{Z})\right)-\tilde{X}\left({ }^{C G} g_{f}(J \tilde{Y}, \tilde{Z})\right) \\
& +{ }^{C G} g_{f}\left(\left(L_{\tilde{Y}} J\right) \tilde{X}, \tilde{Z}\right)+{ }^{C G} g_{f}\left(\tilde{Y},\left(L_{\tilde{Z}} J\right) \tilde{X}\right)
\end{aligned}
$$


for all $\tilde{X}, \tilde{Y}, \tilde{Z} \in \Im_{0}^{1}\left(T^{*} M\right)$. For pairs $\tilde{X}={ }^{H} X,{ }^{V} \omega, \tilde{Y}={ }^{H} Y,{ }^{V} \theta$ and $\widetilde{Z}={ }^{H} Z,{ }^{V} \sigma$, then we get

$$
\begin{aligned}
& \left(\Phi_{J}{ }^{C G} g_{f}\right)\left({ }^{H} X,{ }^{V} \theta,{ }^{H} Z\right)=2^{C G} g_{f}\left({ }^{V} \theta,{ }^{V}(p \circ R(X, Z))\right. \\
& \left(\Phi_{J}{ }^{C G} g_{f}\right)\left({ }^{H} X,{ }^{H} Y,{ }^{V} \sigma\right)=2^{C G} g_{f}\left({ }^{V}\left(p \circ R(X, Y),{ }^{V} \sigma\right) .\right.
\end{aligned}
$$

and the others is zero. Therefore, we have the following result.

Theorem 3. Let $(M, g)$ be a Riemannian manifold and let $T^{*} M$ be its cotangent bundle equipped with the rescaled Cheeger Gromoll type metric ${ }^{C G} g_{f}$ and the paracomplex structure $J$. The triple $\left(T^{*} M, J,{ }^{C G} g_{f}\right)$ is a para-Kähler-Norden (paraholomorphic Norden) manifold if and only if $M$ is flat.

Remark 1. Let $(M, g)$ be a Riemannian manifold and let $T^{*} M$ be its cotangent bundle equipped with the rescaled Cheeger Gromoll type metric ${ }^{C G} g_{f}$. The diagonal lift ${ }^{D} \gamma$ of $\gamma \in \Im_{1}^{1}(M)$ to $T^{*} M$ is defined by the formulas

$$
\begin{aligned}
{ }^{D} \gamma^{H} X & ={ }^{H}(\gamma X) \\
{ }^{D} \gamma^{V} \omega & =-{ }^{V}(\omega \circ \gamma)
\end{aligned}
$$

for any $X \in \Im_{0}^{1}(M)$ and $\omega \in \Im_{1}^{0}(M)$. The diagonal lift ${ }^{D} I$ of the identity tensor field $I \in \Im_{1}^{1}(M)$ has the following properties

$$
\begin{aligned}
{ }^{D} I^{H} X & ={ }^{H} X \\
{ }^{D} I^{V} \omega & =-{ }^{V} \omega
\end{aligned}
$$

and satisfies $\left({ }^{D} I\right)^{2}=I_{T^{*} M}$. Thus, ${ }^{D} I$ is an almost paracomplex structure. Also, the rescaled Cheeger Gromoll type metric ${ }^{C G} g_{f}$ is pure with respect to ${ }^{D} I$, e.i. the triple $\left(T^{*} M,{ }^{D} I,{ }^{C G} g_{f}\right)$ is an almost paracomplex Norden manifold. Finally, by using $\Phi$-operator, we can say that the rescaled Cheeger Gromoll type metric ${ }^{C G} g_{f}$ is paraholomorphic with respect to ${ }^{D} I$ if and only if $M$ is flat.

The following remark follows directly from Proposition 2

Remark 2. The cotangent bundle $\left(T^{*} M,{ }^{C G} g_{f}\right)$ is never flat.

As is known that the almost paracomplex Norden structure is a specialized Riemannian almost product structure on a Riemannian manifold. The theory of Riemannian almost product structures was initiated by K. Yano in [37. The classification of Riemannian almost-product structure with respect to their covariant derivatives is described by A.M. Naveira in [17. This is the analogue of the classification of almost Hermitian structures by A. Gray and L. Hervella in [12. Having in mind these results, M. Staikova and K. Gribachev obtained a classification of the Riemannian almost product structures, for which the trace vanishes (see [30]). There are lots of physical applications involving a Riemannian almost product manifold. Now we shall give some applications for almost paracomplex Norden structures in context of almost product Riemannian manifolds.

4.1. Let us recall almost product Riemannian manifolds. If an $n$-dimensional Riemannian manifold $M$, endowed with a positive definite Riemannian metric $g$, admits a non-trivial tensor field $F$ of type (1.1) such that

$$
F^{2}=I
$$

and

$$
g(F X, Y)=g(X, F Y)
$$


for every vector fields $X, Y \in \Im_{0}^{1}(M)$, then $F$ is called an almost product structure and $(M, F, g)$ is called an almost product Riemannian manifold. An integrable almost product Riemannian manifold with structure tensor $F$ is called a locally product Riemannian manifold. If $F$ is covariantly constant with respect to the Levi-Civita connection $\nabla$ of $g$ which is equivalent to $\Phi_{F} g=0$, then $(M, F, g)$ is called a locally decomposable Riemannian manifold.

Now consider the almost product structure $J$ defined by (4.2) and the LeviCivita connection $\widetilde{\nabla}$ given by Proposition 1, We define a tensor field of type $(1,2)$ on $T^{*} M$ by

$$
\widetilde{S}(\widetilde{X}, \widetilde{Y})=\frac{1}{2}\left\{\left(\widetilde{\nabla}_{J \widetilde{Y}} J\right) \widetilde{X}+J\left(\left(\widetilde{\nabla}_{\widetilde{Y}} J\right) \widetilde{X}\right)-J\left(\left(\widetilde{\nabla}_{\widetilde{X}} J\right) \widetilde{Y}\right)\right\}
$$

for all $\widetilde{X}, \widetilde{Y} \in \Im_{0}^{1}\left(T^{*} M\right)$. Then the linear connection

$$
\bar{\nabla}_{\widetilde{X}} \widetilde{Y}=\widetilde{\nabla}_{\widetilde{X}} \widetilde{Y}-\widetilde{S}(\widetilde{X}, \widetilde{Y})
$$

is an almost product connection on $T^{*} M$ (for almost product connection, see [14]).

Theorem 4. Let $(M, g)$ be a Riemannian manifold and let $T^{*} M$ be its cotangent bundle equipped with the rescaled Cheeger Gromoll type metric ${ }^{C G} g_{f}$ and the almost product structure $J$. Then the almost product connection $\bar{\nabla}$ constructed by the Levi-Civita connection $\widetilde{\nabla}$ of the rescaled Cheeger Gromoll type metric ${ }^{C G} g_{f}$ and the almost product structure $J$ is as follows:

$$
\left\{\begin{aligned}
& \text { i) } \bar{\nabla}_{H X}{ }^{H} Y={ }^{H}\left(\nabla_{X} Y\right)+{ }^{H}\left({ }^{f} A(X, Y)\right) \\
& \text { ii) } \bar{\nabla}_{H_{X}}{ }^{V} \theta={ }^{V}\left(\nabla_{X} \theta\right), \\
& \text { iii) } \bar{\nabla}_{V_{\omega}}{ }^{H} Y= \frac{3}{2 f \alpha}{ }^{H}\left(p\left(g^{-1} \circ R(, Y) \tilde{\omega}\right)\right), \\
& \text { iv) } \bar{\nabla}_{V_{\omega} \omega}{ }^{V} \theta=-\frac{1}{\alpha}\left({ }^{C G} g\left({ }^{V} \omega, \gamma \delta\right){ }^{V} \theta+{ }^{C G} g_{f}\left({ }^{V} \theta, \gamma \delta\right)^{V} \omega\right)+\frac{\alpha+1}{\alpha}{ }^{C G} g_{f}\left({ }^{V} \omega,{ }^{V} \theta\right) \gamma \delta \quad-\frac{1}{\alpha}{ }^{C G} g_{f}\left({ }^{V} \omega, \gamma \delta\right)^{C G} g_{f}\left({ }^{V} \theta, \gamma \delta\right) \gamma \delta .
\end{aligned}\right.
$$

Denoting by $\bar{T}$, the torsion tensor of $\bar{\nabla}$, we have from (4.2), (4.4) and (4.5)

$$
\begin{aligned}
\bar{T}\left({ }^{V} \omega,{ }^{V} \theta\right) & =0, \\
\bar{T}\left({ }^{V} \omega,{ }^{H} Y\right) & =\frac{3}{2 f \alpha}{ }^{H}\left(p\left(g^{-1} \circ R(, Y) \tilde{\omega}\right)\right), \\
\bar{T}\left({ }^{H} X,{ }^{H} Y\right) & =-{ }^{V}(p \circ R(X, Y)) .
\end{aligned}
$$

Hence we have the theorem below

Theorem 5. Let $(M, g)$ be a Riemannian manifold and let $T^{*} M$ be its cotangent bundle. The almost product connection $\bar{\nabla}$ constructed by the Levi-Civita connection $\widetilde{\nabla}$ of the rescaled Cheeger Gromoll type metric ${ }^{C G} g_{f}$ and the almost product structure $J$ is symmetric if and only if $M$ is flat.

As is well-known, if there exists a symmetric almost product connection on $M$ then the almost product structure $J$ is integrable [14. The converse is also true [8]. Thus we get the following conclusion.

Corollary 1. Let $(M, g)$ be a Riemannian manifold and $T^{*} M$ be its tangent bundle equipped with the rescaled Cheeger Gromoll type metric ${ }^{C G} g_{f}$ and the almost product structure $J$. The triple $\left(T^{*} M, J,{ }^{C G} g_{f}\right)$ is a locally product Riemannian manifold if and only if $M$ is flat. 
Similarly, let us consider the almost product structure ${ }^{D} I$ and the Levi-Civita connection $\widetilde{\nabla}$ of the rescaled Cheeger Gromoll type metric ${ }^{C G} g_{f}$, Another almost product connection can be constructed.

If $J$ is covariantly constant with respect to the Levi-Civita connection $\widetilde{\nabla}$ of the rescaled Cheeger Gromoll type metric ${ }^{C G} g_{f}$ which is equivalent to $\Phi_{J}^{C G} g_{f}=0$, then $\left(T^{*} M, J,{ }^{C G} g_{f}\right)$ is called a locally decomposable Riemannian manifold. In view of Theorem 3 , we have the following.

Corollary 2. Let $(M, g)$ be a Riemannian manifold and $T^{*} M$ be its cotangent bundle equipped with the rescaled Cheeger Gromoll type metric ${ }^{C G} g_{f}$ and the almost product structure $J$. The triple $\left(T^{*} M, J,{ }^{C G} g_{f}\right)$ is a locally decomposable Riemannian manifold if and only if $M$ is flat.

4.2. Let $\left(M_{2 k}, \varphi, g\right)$ be a non-integrable almost paracomplex manifold with a Norden metric. An almost paracomplex Norden manifold $\left(M_{2 k}, \varphi, g\right)$ is a quasipara-Kähler-Norden manifold, if $\underset{X, Y, Z}{\sigma} g\left(\left(\nabla_{X} \varphi\right) Y, Z\right)=0$, where $\sigma$ is the cyclic sum by three arguments [15]. In [28], the authors proved that $\underset{X, Y, Z}{\sigma} g\left(\left(\nabla_{X} \varphi\right) Y, Z\right)=0$ is equivalent to $\left(\Phi_{\varphi} g\right)(X, Y, Z)+\left(\Phi_{\varphi} g\right)(Y, Z, X)+\left(\Phi_{\varphi} g\right)(Z, X, Y)=0$. We compute

$$
A(\tilde{X}, \tilde{Y}, \tilde{Z})=\left(\Phi_{J}^{C G} g_{f}\right)(\tilde{X}, \tilde{Y}, \tilde{Z})+\left(\Phi_{J}{ }^{C G} g_{f}\right)(\tilde{Y}, \tilde{Z}, \tilde{X})+\left(\Phi_{J}{ }^{C G} g_{f}\right)(\tilde{Z}, \tilde{X}, \tilde{Y})
$$

for all $\tilde{X}, \tilde{Y}, \widetilde{Z} \in \Im_{0}^{1}\left(T^{*} M\right)$. By means of (4.3), we have $A(\tilde{X}, \tilde{Y}, \tilde{Z})=0$ for all $\widetilde{X}, \widetilde{Y}, \widetilde{Z} \in \Im_{0}^{1}\left(T^{*} M\right)$. Hence we state the following theorem.

Theorem 6. Let $(M, g)$ be a Riemannian manifold and $T^{*} M$ be its cotangent bundle equipped with the rescaled Cheeger Gromoll type metric ${ }^{C G} g_{f}$ and the almost paracomplex structure $J$ defined by (4.2). The triple $\left(T^{*} M, J,{ }^{C G} g_{f}\right)$ is a quasi-paraKähler-Norden manifold.

O. Gil-Medrano and A.M. Naveira proved that both distributions of the almost product structure on the Riemannian manifold $(M, F, g)$ are totally geodesic if and only if $\underset{X, Y, Z}{\sigma} g\left(\left(\nabla_{X} \varphi\right) Y, Z\right)=0$ for any $X, Y, Z \in \Im_{0}^{1}(M)$ [1]. As a consequence of Theorem 6, we have the following.

Corollary 3. Both distributions of the almost product Riemannian manifold $\left(T^{*} M\right.$, $\left.J,{ }^{C G} g_{f}\right)$ are totally geodesic.

4.3. Let $F$ be an almost product structure and $\nabla$ be a linear connection on an $n$-dimensional Riemannian manifold $M$. The product conjugate connection $\nabla^{(F)}$ of $\nabla$ is defined by

$$
\nabla_{X}^{(F)} Y=F\left(\nabla_{X} F Y\right)
$$

for all $X, Y \in \Im_{0}^{1}(M)$. If $(M, F, g)$ is an almost product Riemannian manifold, then $\left(\nabla_{X}^{(F)} g\right)(F Y, F Z)=\left(\nabla_{X} g\right)(Y, Z)$, i.e. $\nabla$ is a metric connection with respect to $g$ if and only if $\nabla^{(F)}$ is so. From this, we can say that if $\nabla$ is the Levi-Civita connection of $g$, then $\nabla^{(F)}$ is a metric connection with respect to $g$ [3]. The family of metric connections on a Riemannian manifold $M$ which have the same geodesics as the Levi-Civita connection is a distinguished class among the family of all connections on $M$. This family attracted the attention of E. Cartan in the early 20th century. Since then, many mathematicians have been concerned with its study. These connections arise in a natural way in theoretical and mathematical physics. For 
example, such a connection is of particular interest in string and superstring theory [1, 31.

By the almost product structure $J$ defined by (4.2) and the Levi-Civita connection $\widetilde{\nabla}$ given by Proposition 1, we write the product conjugate connection $\widetilde{\nabla}^{(J)}$ of $\widetilde{\nabla}$ as follows:

$$
\widetilde{\nabla}_{\widetilde{X}}^{(J)} \widetilde{Y}=J\left(\widetilde{\nabla}_{\widetilde{X}} J \widetilde{Y}\right)
$$

for all $\widetilde{X}, \tilde{Y} \in \Im_{0}^{1}\left(T^{*} M\right)$. Also note that $\widetilde{\nabla}^{(J)}$ is a metric connection of the rescaled Cheeger Gromoll type metric ${ }^{C G} g_{f}$. The standart calculations give the following theorem.

Theorem 7. Let $(M, g)$ be a Riemannian manifold and let $T^{*} M$ be its cotangent bundle equipped with the rescaled Cheeger Gromoll type metric ${ }^{C G} g_{f}$ and the almost product structure $J$. Then the product conjugate connection (or metric connection) $\widetilde{\nabla}^{(J)}$ is as follows:

i) $\widetilde{\nabla}_{{ }^{H}}{ }^{H} Y={ }^{H}\left(\nabla_{X} Y+{ }^{f} A(X, Y)\right)-\frac{1}{2}^{V}(p \circ R(X, Y))$,

ii) $\widetilde{\nabla}_{H}{ }^{V} \theta=-\frac{1}{2 f \alpha}{ }^{H}\left(p\left(g^{-1} \circ R(, X) \widetilde{\theta}\right)\right)+{ }^{V}\left(\nabla_{X} \theta\right)$,

iii) $\widetilde{\nabla}_{V_{\omega}}{ }^{H} Y=\frac{1}{2 f \alpha}^{H}\left(p\left(g^{-1} \circ R(, Y) \tilde{\omega}\right)\right)$,

iv) $\widetilde{\nabla}_{{ } \omega}{ }^{V} \theta=-\frac{1}{\alpha}\left({ }^{C G} g\left({ }^{V} \omega, \gamma \delta\right){ }^{V} \theta+{ }^{C G} g_{f}\left({ }^{V} \theta, \gamma \delta\right){ }^{V} \omega\right)+\frac{\alpha+1}{\alpha}{ }^{C G} g_{f}\left({ }^{V} \omega,{ }^{V} \theta\right) \gamma \delta$ $-\frac{1}{\alpha}{ }^{C G} g_{f}\left({ }^{V} \omega, \gamma \delta\right)^{C G} g_{f}\left({ }^{V} \theta, \gamma \delta\right) \gamma \delta$.

The relationship between curvature tensors $R_{\nabla}$ and $R_{\nabla_{(F)}}$ of the connections $\nabla$ and $\nabla^{(F)}$ is follows: $R_{\nabla^{(F)}}(X, Y, Z)=F\left(R_{\nabla}(X, Y, F Z)\right.$ for all $X, Y, Z \in \Im_{0}^{1}(M)$ 3. By means of the almost product structure $J$ defined by (4.2) and Proposition 2, From $\widetilde{R}_{\widetilde{\nabla}^{(J)}}(\widetilde{X}, \widetilde{Y}, \widetilde{Z})=J\left(\widetilde{R}_{\widetilde{\nabla}}(\widetilde{X}, \widetilde{Y}, J \widetilde{Z})\right.$, components of the curvature tensor $\widetilde{R}_{\widetilde{\nabla}^{(J)}}$ of the product conjugate connection (or metric connection) $\widetilde{\nabla}^{(J)}$ can easily be computed. Lastly, by using the almost product structure ${ }^{D} I$, another metric connection of the rescaled Cheeger Gromoll type metric ${ }^{C G} g_{f}$ can be constructed.

\section{REFERENCES}

[1] I. Agricola, The Srni lectures on non-integrable geometries with torsion. Arch. Math. (Brno) 42 (2006), no. 5, 5-84.

[2] F. Agca, A. A. Salimov, Some notes concerning Cheeger-Gromoll metrics. Hacet. J. Math. Stat. to appear

[3] A. M. Blaga, M. Crasmareanu, The geometry of product conjugate connections. An. Stiint. Univ. Al. I. Cuza Iasi. Mat. (N.S.), 59(2013), no. 1, 73-84.

[4] J. Cheeger, D. Gromoll, On the structure of complete manifolds of nonnegative curvature. Ann. of Math., 96 (1972), 413-443.

[5] V. Cruceanu, P. Fortuny, P. M. Gadea, A survey on paracomplex Geometry. Rocky Mountain J. Math., 26 (1995), 83-115.

[6] V. Cruceanu, Une classe de structures géométriques sur le fibré cotangent. Tensor (N.S.) 53 (1993), Commemoration Volume I, 196-201.

[7] L. S. Druţă, Classes of general natural almost anti-Hermitian structures on the cotangent bundles. Mediterr. J. Math. 8 (2011), no. 2, 161-179.

[8] A. Fujimoto, Theory of G-structures. Publ. Study Group of Geometry, 1, Tokyo Univ., Tokyo, 1972.

[9] A. Gezer, M. Altunbas, Some notes concerning Riemannian metrics of Cheeger Gromoll type. J. Math. Anal. Appl. 396 (2012), no. 1, 119-132.

[10] A. Gezer, M. Altunbas, Notes on the rescaled Sasaki type metric on the cotangent bundle. Acta Math. Sci. Ser. B Engl. Ed. to appear. 
[11] O. Gil-Medrano and A. M. Naveira, Some remarks about the Riemannian curvature operator of a Riemannian almost-product manifold. Rev. Roum. Math. Pures Appl. 30(18) (1985) $647-658$.

[12] A. Gray, L.M. Hervella, The sixteen classes of almost Hermitian manifolds and their linear invariants. Ann. Mat. Pura Appl., IV. Ser. 123 (1980), pp. 35-58.

[13] G. I. Kruchkovich, Hypercomplex structure on manifold. I, Tr. Sem. Vect. Tens. Anal, Moscow Univ., 16 (1972), 174-201.

[14] M. de Leon, P. R. Rodrigues, Methods of Differential Geometry in Analytical Mechanics. North-Holland Mathematics Studies, 1989.

[15] M. Manev and D. Mekerov, On Lie groups as quasi-K“"ahler manifolds with Killing Norden metric. Adv. Geom. 8(3) (2008) 343-352.

[16] E. Musso, F. Tricerri, Riemannian Metrics on Tangent Bundles. Ann. Mat. Pura. Appl., 150 (1988), no. 4, 1-19.

[17] A.M. Naveira, A classification of Riemannian almost-product manifolds. Rend. Mat.Appl., VII. Ser. 3 (1983), pp. 577-592..

[18] Z. Olszak, On almost complex structures with Norden metrics on tangent bundles. Period. Math. Hungar. 51 (2005), no. 2, 59-74.

[19] V. Oproiu, N. Papaghiuc, Einstein quasi-anti-Hermitian structures on the tangent bundle. An. Ştiinţ. Univ. Al. I. Cuza Iaşi. Mat. (N.S.) 50 (2004), no. 2, 347-360

[20] V. Oproiu, N. Papaghiuc, Classes of almost anti-Hermitian structures on the tangent bundle of a Riemannian manifold. An. Ştiinţ. Univ. Al. I. Cuza Iaşi. Mat. (N.S.) 50 (2004), no. 1, $175-190$.

[21] V. Oproiu, N. Papaghiuc, Some classes of almost anti-Hermitian structures on the tangent bundle. Mediterr. J. Math. 1 (2004), no. 3, 269-282.

[22] V. Oproiu, N. Papaghiuc, Some examples of almost complex manifolds with Norden metric. Publ. Math. Debrecen 41 (1992), no. 3-4, 199-211.

[23] N. Papaghiuc, A locally symmetric complex structure with Norden metric on the tangent bundle of a space form. An. Ştiinţ. Univ. Al. I. Cuza Iaşi. Mat. (N.S.) 48 (2002), no. 1, $101-112$

[24] N. Papaghiuc, Some almost complex structures with Norden metric on the tangent bundle of a space form. An. Ştiinţ. Univ. Al. I. Cuza Iaşi. Mat. (N.S.) 46 (2000), no. 1, 99-110

[25] A. A. Salimov, M. Iscan, F. Etayo, Paraholomorphic B-manifold and its properties. Topology Appl., 154 (2007), no. 4, 925-933.

[26] A. Salimov, A. Gezer, M. Iscan, On para-Kähler-Norden structures on the tangent bundles. Ann. Polon. Math. 103 (2012), no. 3, 247-261.

[27] A. Salimov, A. Gezer, On the geometry of the (1,1) -tensor bundle with Sasaki type metric. Chin. Ann. Math. Ser. B 32 (2011), no. 3, 369-386.

[28] A.A. Salimov, M. Iscan, K. Akbulut, Notes on para-Norden-Walker 4-manifolds. Int. J. Geom. Methods Mod. Phys. 7 (2010), no. 8, 1331-1347.

[29] S. Sasaki, On the differential geometry of tangent bundles of Riemannian manifolds. Tohoku Math. J., 10 (1958) 338-358.

[30] M.T. Staikova, K.I. Gribachev, Canonical connections and their conformal invariants on Riemannian almost-product manifolds. Serdica 18 (1992), no. 3-4, 150-161.

[31] A. Strominger, Superstrings with torsion. Nucl. Phys. B 274 (1986), 253-284.

[32] S. Tachibana, Analytic tensor and its generalization. Tohoku Math. J., 12 (1960), no.2, 208221.

[33] V. V. Vishnevskii, Integrable affinor structures and their plural interpretations. J. of Math. Sciences, 108 (2002), no. 2, 151-187.

[34] V.V. Vishnevskii, A. P. Shirokov, V. V. Shurygin, Spaces over algebras. Kazan Gos. University, Kazan, 1985 (Russian).

[35] K. Yano, M. Ako, On certain operators associated with tensor field. Kodai Math. Sem. Rep., 20 (1968), 414-436.

[36] K. Yano, S. Ishihara, Tangent and Cotangent Bundles. Marcel Dekker, Inc., New York 1973.

[37] K. Yano, Differential geometry on complex and almost complex spaces. Pure and Applied Math. vol. 49, New York, Pergamon Press Book, 1965.

[38] B. V. Zayatuev, On geometry of tangent Hermitian surface. Webs and Quasigroups. T.S.U. (1995), 139-143. 
[39] B. V. Zayatuev, On some clases of AH-structures on tangent bundles. Proceedings of the International Conference dedicated to A. Z. Petrov [in Russian], 2000, pp. 53-54.

[40] B. V. Zayatuev, On some classes of almost-Hermitian structures on the tangent bundle. Webs and Quasigroups. T.S.U. (2002), 103-106.

Ataturk University, Faculty of Science, Department of Mathematics, 25240, ErzurumTURKEY.

E-mail address: agezer@atauni.edu.tr

Erzincan University, Faculty of Science and Art, Department of Mathematics, 24030, ERZINCAN-TURKEY.

E-mail address: maltunbas@erzincan.edu.tr 